\title{
Caracterizaçáo clínica e motora-funcional de idosos hospitalizados pós-Acidente Vascular Cerebral
}

\author{
Clinical and motor functional of hospitalized elderly after Stroke
}

\author{
Maria Clarice Lopes da Silva', Janaine Cunha Polese ${ }^{2}$, Juliana Maria Pimenta \\ Starling ${ }^{3}$, Leani Souza Máximo Pereira ${ }^{4}$
}

\section{RESUMO}

Objetivo. Caracterizar uma amostra de idosos pós Acidente Vascular Cerebral (AVC) agudo internados em uma unidade de AVC de um hospital universitário quanto a variáveis clínicas e motora-funcionais, bem como verificar possíveis ganhos motores durante o período de internação. Método. Estudo observacional retrospectivo com coleta de dados a partir de fichas de avaliação padronizadas do setor de fisioterapia. Foram coletados o sexo e idade dos pacientes, além de variáveis clínicas e motora-funcionais. Estatísticas descritivas para caracterização da amostra foram realizadas. Resultados. De um total de 349 idosos que passaram pela unidade entre maio de 2012 a agosto de $2013,52 \%$ eram homens, a média de idade foi de $73 \pm 8,1$ anos, sendo que $85 \%$ sofreram AVC isquêmico. Os pacientes receberam uma média de $8 \pm 9,6$ sessóes de fisioterapia e foi observado aumento percentual nas variáveis motora- funcionais: controle de tronco sem apoio, equilíbrio estático bom e deambulação sem apoio no momento da avaliação fisioterápica final em comparação à avaliação inicial (aumentos em 13\%, 12\% e 13\%, respectivamente). Conclusóes. A identificação clínica e motora-funcional de indivíduos idosos em uma unidade de AVC pode possibilitar o planejamento e implantação de estratégias de reabilitação eficazes tanto na internação quanto após a alta hospitalar.

Unitermos. Acidente Vascular Cerebral, Fisioterapia, Idosos, Hospitalização

Citaçáo. Silva MCL, Polese JC, Starling JMP, Pereira LSM. Caracterização clínica e motora-funcional de idosos hospitalizados pós-Acidente Vascular Cerebral.

Trabalho realizado no Hospital Risoleta Tolentino Neves da Universidade Federal de Minas Gerais - UFMG, Belo Horizonte-MG, Brasil.

\footnotetext{
1.Fisioterapeuta, Residência Multiprofissional em Saúde do Idoso do Hospital das Clínicas da Universidade Federal de Minas Gerais - UFMG, Belo Horizonte-MG, Brasil.

2.Fisioterapeuta, Doutoranda em Ciências da Reabilitação, Universidade Federal de Minas Gerais - UFMG, Belo Horizonte-MG, Brasil. Ph.D Candidate in Health Sciences, Universityof Sydney, Sydney, New South Wales, Australia. 3.Fisioterapeuta, Mestranda em Ciências da Reabilitaçáo, Universidade Federal de Minas Gerais - UFMG, Belo Horizonte-MG, Brasil.

4.Fisioterapeuta, Doutora, Professora Adjunta do Departamento de Fisioterapia da Universidade Federal de Minas Gerais - UFMG, Belo Horizonte-MG, Brasil.
}

\section{ABSTRACT}

Aim. To characterize a sample of elderly after acute stroke admitted to a stroke unit of a university hospital regarding clinical, functional, and motor variables and check possible motor gains during the period of hospitalization. Method. An exploratory cross-sectional study was conducted with data collection from the physical therapy unit assessment standardized files. Sex and age were collected, in addition to clinical and functional and motor variables. In order to characterize the sample, descriptive statistics were performed. Results. A total of 349 elderly post stroke that went through the unit from May 2012 to August 2013, $52 \%$ were men, with an average age of $73 \pm 8.1$ years, and $85 \%$ suffered ischemic stroke. The average number of physiotherapy sessions received by patients was 8 sessions. There were gains in the percentage values of the variables: trunk control without support, good static balance and ambulation without support for the final physical therapy evaluation compared to baseline (increases by $13 \%$, $12 \%$ and $13 \%$, respectively). Conclusions. The clinical and motor -functional identification of elderly individuals in a stroke unit can enable the planning and implementation of effective rehabilitation strategies at admission and after discharge.

Keywords. Stroke, Physical Therapy, Aged, Hospitalization

Citation. Silva MCL, Polese JC, Starling JMP, Pereira LSM.Clinical and motor functional of hospitalized elderly after Stroke
Endereço para correspondência: Leani Souza Máximo Pereira Departamento de Fisioterapia - UFMG Av. Antônio Carlos, 6627, Campus Pampulha CEP 31270-901 Belo Horizonte-MG, Brasil Fone: 55-31-3409-173-95 Fax: 55-31-3409-4781 E-mail:leanismp.bh@terra.com.br; mcla.lopes@yahoo.com.br Aceito em: 08/08/14

Conflito de interesses: não 


\section{INTRODUÇÃO}

O crescente envelhecimento populacional pelo qual vem passando os países em desenvolvimento, no qual se inclui o Brasil, coloca em evidência uma notória incidência de doenças que atingem principalmente a população idosa, dentre elas, as doenças cerebrovasculares, destacando-se o Acidente Vascular Cerebral (AVC) como a sendo mais frequente ${ }^{1}$. Sabe-se que há um aumento da sua incidência a partir da sexta década de vida, praticamente dobrando a relação a cada nova década ${ }^{2}$.

O AVC pode causar deficiências graves que afetam a estrutura corporal, podendo impactar na funcionalidade do indivíduo ${ }^{3,4}$. No idoso em especial, a presença de incapacidades apresentadas pós-AVC agudo, associadas ou não a alterações fisiológicas decorrentes do próprio processo de envelhecimento podem influenciar negativamente na reabilitação 5 . A habilidade de alinhar o tronco e manter-se sentado e a capacidade de permanecer em ortostatismo com um bom equilíbrio e deambular, por exemplo, podem tornar-se aquisições mais difíceis pós-AVC, principalmente ao indivíduo idoso ${ }^{5,6}$. Diversos estudos vêm colocando em evidência fatores que são preditivos de piores resultados funcionais pós-AVC, dentre eles: a diminuição acentuada ou ausência de movimentação e uso do membro superior e/ou inferior acometido, a perda de equilíbrio e coordenação na posição sentado e em ortostatismo, além da idade avançada ${ }^{7-11}$.

A avaliação e identificação dos déficits e incapacidades na fase aguda do AVC devem começar desde a fase de internação hospitalar ${ }^{12}$. Nesse contexto, ressalta-se a importância das Unidades de AVC hospitalares, que contam com uma equipe multidisciplinar especialmente capacitada no atendimento dessa condição de saúde ${ }^{13}$. A avaliação clínica e motora-funcional dos indivíduos, especialmente idosos, ainda na fase hospitalar possibilita o planejamento e implantação de estratégias de reabilitação eficazes e direcionadas, além de promover a otimização dos serviços oferecidos ${ }^{13,14}$. Quanto mais precocemente condições clínicas e motora-funcionais passíveis de intervenção puderem ser identificados, melhor poderá ser o prognóstico terapêutico e a probabilidade de recuperação do individuo ${ }^{10,15}$. Diante do exposto, o objetivo desse estudo foi caracterizar uma amostra de idosos pós AVC agudo internados em uma Unidade de AVC de um hos- pital universitário quanto a variáveis demográficas, clínicas e motora-funcionais, bem como verificar possíveis ganhos motores durante o período de internação.

\section{MÉTODO}

\section{Amostra}

Foi realizado um estudo observacional retrospectivo com coleta de dados realizado a partir de fichas de avaliação padronizadas criadas pelo setor de fisioterapia da Unidade de AVC do Hospital Risoleta Tolentino Neves, em Belo Horizonte.

A amostra foi composta por pacientes idosos internados na unidade de AVC do próprio hospital no período de maio de 2012 a agosto de 2013, de acordo com os seguintes critérios de inclusão: (1) acometimento por AVC ou Ataque Isquêmico Transitório (AIT) diagnosticado pelo médico, (2) indivíduos que tivessem idade igual ou superior a 60 anos. Foram excluídos do estudo indíviduos internados por diagnóstico de outras condiçôes de saúde que não o AVC, além de pacientes que não puderam ser avaliados durante todo o período de internação devido instabilidade clínica limitante ou quadro confusional agudo. O estudo foi aprovado pelo Comitê de Ética em Pesquisa da Universidade Federal de Minas Gerais, parecer no 414.149.

\section{Procedimento}

Para caracterização da amostra foram coletados a idade, sexo, além de variáveis clínicas e motora-funcionais contidos na ficha de avaliação padronizada criada pelo serviço de fisioterapia da unidade de AVC do Hospital Risoleta Tolentino Neves e que eram preenchidas pelos próprios fisioterapeutas, os quais passavam por um treinamento prévio antes de começarem a atuar no setor.

\section{Variáveis Clinicas}

Foram analisados: (1) tipo de AVC (isquêmico, hemorrágico ou AIT), (2) realização ou não de trombólise, (3) lado do hemicorpo acometido (sem acometimento, direito, esquerdo ou ambos), (4) a necessidade de ventilação mecânica com tubo orotraqueal, ventilação não invasiva e/ou aspiração nasotraqueal e (5) o desfecho final do paciente (se alta hospitalar, alta fisioterapêutica, transferência de enfermaria, transferência de instituição, Cen- 
tro de Terapia Intensiva (CTI) ou óbito). Foi registrado ainda o tempo entre a data do ictus e a data da admissão hospitalar, o número de dias em acompanhamento com a fisioterapia e a quantidade de atendimentos fisioterápicos (sessões) recebidos pelos pacientes da amostra.

\section{Variáveis Motora-Funcionais}

As variáveis motora-funcionais dos indivíduos participantes foram avaliados e classificados da seguinte forma:

- Acometimento do membro superior e do membro inferior: se (1) plégico, (2) parético, (3) dismétrico ou (4) sem déficit motor.

- Controle de Tronco: avaliado como a capacidade do indivíduo em manter-se assentado a beira do leito por no mínimo 60 segundos. Capacidade de manter-se sentado pelo tempo estabelecido sem nenhum suporte no tronco era dado como controle de tronco sem apoio (1) e necessidade de algum tipo de suporte externo no tronco era dado como com apoio (2). Controle de tronco não avaliado/realizado (3) era classificado pela impossibilidade de se ficar assentado pelo tempo estabelecido ou devido alguma instabilidade clínica que impedisse a realização do teste.

- Equilíbrio estático: (1) equilíbrio bom era dado pela capacidade do indivíduo manter-se em ortostatismo por um minuto sem apoio e em posição tandem por 10 segundos. (2) equilíbrio regular como a capacidade do indivíduo manter-se em ortostatismo por um minuto sem apoio, porém incapaz de ficar em posição tandem por 10 segundos. (3) equilíbrio ruim foi classificado quando o indivíduo não conseguia manter-se em ortostatismo por um minuto e/ou necessariamente necessitava de auxílio externo (segurar em uma pessoa ou em um dispositivo de auxílio). Impossibilidade de realização do teste foi considerado como (4) não avaliado/realizado.

- Deambulação: avaliado com o indivíduo percorrendo uma distância de 10 metros em um corredor com velocidade usual e classificada: (1) sem apoio - quando o individuo foi capaz de caminhar sem qualquer auxílio externo, com bom equilíbrio, (2) apoio único - necessidade de deambular com apoio unilateral, como o uso de uma bengala, muleta ou auxílio unilateral de uma pessoa, (3) apoio duplo - quando o paciente necessitava de apoio externo bilateral para deambular, e, por fim, (4) não avaliado/realizado quando não era possível se fazer a avaliação da marcha.

Todas as variáveis motoras eram avaliadas e registradas no momento da Avaliação I (avaliação fisioterápica inicial realizada no dia da admissão do paciente na unidade de AVC) e na Avaliação II (avaliação fisioterápica final correspondente ao dia do desfecho final do paciente), e foram utilizadas no presente estudo para comparação dos resultados entre os dois momentos.

\section{Análise Estatística}

Foram utilizadas estatísticas descritivas para caracterização da amostra. Os dados são apresentados como medidas de tendência central (média e desvio padrão) para as variáveis quantitativas, e medidas de frequência e porcentagem para as variáveis categóricas. Todas as análises foram realizadas utilizando o programa estatístico SPSS para Windows versão 17.0.

\section{RESULTADOS}

Durante o período de um ano e três meses foram internados na Unidade de AVC do HRTN 349 idosos, com uma média de idade de $73 \pm 8,1$ anos. Do total da amostra, 52\% dos indivíduos eram do sexo masculino $(n=183)$ e $48 \%$ do sexo feminino $(n=166)$.

\section{Variáveis Clínicas}

Em relação ao tipo de AVC, 85\% (n=295) tiveram o evento isquêmico e apenas $7 \%$ o hemorrágico $(n=25)$. O AIT foi constatado em 29 pacientes correspondendo a $8 \%$ da amostra. Quanto ao tempo de tratamento fisioterápico, foi observada uma média de $7 \pm 7,5$ dias e o número médio de atendimentos de fisioterapia correspondeu a $8 \pm 9,6$ sessóes. A grande maioria dos pacientes - 93\% não precisaram passar por nenhum procedimento como ventilação mecânica, VNI ou aspiração nasotraqueal. O número de óbitos na unidade foi de apenas $2 \%$ sendo que $4 \%$ dos pacientes tiveram de ser transferidos para o CTI devido complicaçóes clínicas. A síntese dos dados clínicos é apresentada na Tabela 1.

\section{Variáveis Motora-Funcionais}

Com relação às variáveis motora-funcionais ana- 
Tabela 1. Caracterização demográfica e clínica da amostra (n=349).

\begin{tabular}{lccc}
\hline Variáveis Clínicas & $\begin{array}{c}\text { Valores } \\
\text { Mínimo }\end{array}$ & $\begin{array}{c}\text { Valores } \\
\text { Máximo }\end{array}$ & Média \pm DP \\
\hline Idade & 60 & 96 & $73 \pm 8,1$ \\
$\begin{array}{l}\text { Intervalo Ictus-admis- } \\
\text { são }\end{array}$ & 0 & 18 & $1 \pm 2,2$ \\
$\begin{array}{l}\text { Número de dias } \\
\text { (acompanhamento }\end{array}$ & 1 & 43 & $7 \pm 7,5$ \\
$\begin{array}{l}\text { fisioterapia) } \\
\text { Número de atendimen- } \\
\text { tos (fisioterapia) }\end{array}$ & 1 & 62 & $8 \pm 9,6$ \\
\hline
\end{tabular}

\begin{tabular}{lcc} 
Variáveis Clínicas & Frequência & $\%$ \\
\hline SEXO & 166 & 48 \\
Feminino & 183 & 52 \\
Masculino & & \\
\hline TIPO DE AVC & 295 & 85 \\
Isquêmico & 25 & 7 \\
Hemorrágico & 29 & 8 \\
Ataque Isquêmico Tran- & \\
sitório &
\end{tabular}

LADO ACOMETIDO

\begin{tabular}{lcc} 
Nenhum & 20 & 6 \\
Direito & 168 & 48 \\
Esquerdo & 149 & 43 \\
Direito e Esquerdo & 12 & 3 \\
\hline
\end{tabular}

\section{TROMBÓLISE}

\begin{tabular}{lcc} 
Não & 333 & 95 \\
Sim & 16 & 5 \\
\hline
\end{tabular}

\section{PROCEDIMENTOS}

\begin{tabular}{lcc} 
Nenhum & 325 & 93 \\
Ventilação Mecânica & 9 & 2,6 \\
Ventilação não-invasiva & 1 & 0,4 \\
Aspiração Nasotraqueal & 14 & 4 \\
\hline DESFECHO & 168 & 48 \\
Alta Hospitalar & 141 & 41 \\
Alta Fisioterapêutica & 15 & 4 \\
CTI & 5 & 2 \\
$\begin{array}{l}\text { Transferência de Insti- } \\
\text { tuição }\end{array}$ & 12 & 3 \\
Transferência de enfer- \\
maria
\end{tabular}

CTI: Centro de Terapia Intensiva, $\mathrm{DP}=$ desvio padrão, $\%=$ porcentagem o membro superior classificado como sem déficit motor na avaliação fisioterápica inicial, sendo que esse valor aumentou para 39\% após a avaliação fisioterápica final. Concomitantemente os que tiveram o membro inferior classificado como sem déficit motor passou de 37\% para $43 \%$, entre a avaliação inicial e final. Os pacientes idosos que possuíam controle de tronco sem apoio no momento da avaliação I eram de $70 \%$, passando para $83 \%$ na avaliação II, havendo um ganho percentual em 13\% para essa variável. Houve ganhos também nos valores encontrados para a variável equilíbrio estático, com um aumento percentual em $12 \%$ dos que tiveram essa condição motora classificada como boa entre a avaliação I e II. Já a quantidade de idosos que deambularam sem apoio passou de $34 \%$ na avaliação inicial para $47 \%$ na avaliação final, com um ganho percentual em 13\%. Os dados correspondentes às variáveis motora-funcionais podem ser observados na Tabela 2 .

\section{DISCUSSÂO}

Este estudo caracterizou uma amostra de indivíduos idosos internados em uma unidade de AVC de um hospital universitário. A proporçáo de idosos acometidos pelo AVC foi relativamente proporcional em ambos os sexos, sendo $48 \%$ mulheres e $52 \%$ homens. O tipo isquêmico foi o mais prevalente. Foram observados aumento em valores percentuais na maioria das variáveis motora-funcionais analisadas entre o momento da avaliação fisioterápica inicial para a avaliação final.

De acordo com dados da literatura, a frequência de AVC é maior em homens do que nas mulheres até os 55 anos e, após essa idade, o risco de ocorrência do evento cerebrovascular aumenta e sua frequência é praticamente igual em ambos os sexos16. Além disso, o AVC isquêmico é responsável por grande parte dos acometimentos, com uma média de prevalência de 80 a $85 \%$ do total de $\operatorname{casos}^{1,14,16}$. Os achados do presente estudo corroboram os dados da literatura, sendo que a média de idade dos pacientes internados foi de 73 anos e a proporção de idosos acometidos foi praticamente simétrica em ambos os sexos, com uma ligeira prevalência no sexo masculino. Com relação ao tipo de AVC $85 \%$ da amostra tiveram o 
Tabela 2. Caracterizaçâo motora-funcional da amostra $(n=349)$.

\begin{tabular}{|c|c|c|c|c|}
\hline \multirow{2}{*}{ Variáveis Motoras } & \multicolumn{2}{|c|}{ Primeira Avaliaçáo } & \multicolumn{2}{|c|}{ Segunda Avaliaçáo } \\
\hline & Frequência & Porcentagem & Frequência & Porcentagem \\
\hline \multicolumn{5}{|l|}{ MEMBRO SUPERIOR } \\
\hline Plégico & 79 & 23 & 65 & 19 \\
\hline Parético & 148 & 42 & 143 & 41 \\
\hline Dismétrico & 5 & 1 & 5 & 1 \\
\hline Sem déficit motor & 117 & 34 & 136 & 39 \\
\hline \multicolumn{5}{|l|}{ MEMBRO INFERIOR } \\
\hline Plégico & 51 & 15 & 41 & 12 \\
\hline Parético & 164 & 47 & 153 & 44 \\
\hline Dismétrico & 4 & 1 & 4 & 1 \\
\hline Sem déficit motor & 130 & 37 & 151 & 43 \\
\hline \multicolumn{5}{|l|}{ CONTROLE DE TRONCO } \\
\hline Não avaliado/realizado & 64 & 18 & 21 & 6 \\
\hline Com apoio & 40 & 12 & 38 & 11 \\
\hline Sem apoio & 245 & 70 & 290 & 83 \\
\hline \multicolumn{5}{|l|}{ EQUILÍBRIO ESTÁTICO } \\
\hline Não avaliado/realizado & 132 & 38 & 69 & 20 \\
\hline Ruim & 18 & 5 & 18 & 5 \\
\hline Regular & 53 & 15 & 74 & 21 \\
\hline Bom & 146 & 42 & 188 & 54 \\
\hline \multicolumn{5}{|l|}{ DEAMBULAÇÁO } \\
\hline Não avaliado/realizado & 165 & 47 & 106 & 30 \\
\hline Apoio duplo & 19 & 5 & 26 & 7 \\
\hline Apoio Único & 47 & 14 & 55 & 16 \\
\hline Sem apoio & 118 & 34 & 162 & 47 \\
\hline
\end{tabular}

evento isquêmico. Em um estudo de caracterização de internações hospitalares por AVC realizado no Sul do Brasil, observou-se também uma ligeira prevalência do AVC em homens, sendo 53,6\% da amostra, e o AVC isquêmico sendo responsável por $83,1 \%$ dos $\operatorname{casos}^{17}$. A faixa etária da maioria dos pacientes da pesquisa estava entre 70 a 79 anos de idade. Já em um estudo epidemiológico sobre o AVC em 19 hospitais de Fortaleza, na região Nordeste do Brasil, encontrou uma prevalência do AVC ligeiramente maior na população feminina, com $51,8 \%$ da amostra estudada, sendo o tipo isquêmico o mais prevalente com $72,9 \%$ do total de acometimentos e uma média de idade dos pacientes de $67,7 \operatorname{anos}^{18}$. médio entre o ictus até a admissão hospitalar foi de um dia. Já em um estudo prévio em hospitais de Fortaleza observou-se um intervalo de 12,9 horas entre o início dos sintomas e a hospitalização dos pacientes da amostra18. Sabe-se que o tempo entre o início dos sintomas e o adequado atendimento especializado deve ser o mais precoce possível para evitar complicaçóes e otimizar o tratamento específico do $\mathrm{AVC}^{19}$.

Os dados encontrados sugerem que talvez ainda seja preciso conscientizar mais a população da importância de se procurar o quanto antes um serviço hospitalar adequado diante do início e identificação de alteraçôes clínicas sintomatológicas causadas pelo AVC. 
Verificou-se também nesse estudo que o tempo Foram observados no presente estudo que a porcentagem de indivíduos que estavam sem déficit no membro superior aumentou em 5\% do momento da avaliação inicial para a avaliação final e em $6 \%$ para o membro inferior. Houve ainda aumento nos valores percentuais em três variáveis motora-funcionais analisadas e suas subclassificações: controle de tronco sem apoio, equilíbrio estático bom e deambulação sem apoio (aumento de $13 \%, 12 \%$ e $13 \%$ na avaliação fisioterápica final em comparação à inicial, respectivamente). Esses resultados podem reforçar a importância da reabilitação o mais precoce possível para os indivíduos idosos pós-AVC agudo, com o objetivo de se ganhar mobilidade e independência, principalmente através do treino de tarefas específicas que demandem equilíbrio, associadas com exercícios de fortalecimento ${ }^{16,20-22}$. Sabe-se que no AVC, as reaçóes de equilíbrio podem estar comprometidas, e com isso, alteraçôes na estabilidade podem ser observadas nas posiçôes sentada e em pé, podendo interferir na funcionalidade do indivíduo ${ }^{6,8,21}$. No paciente idoso, em especial, esse comprometimento já pode estar presente mesmo antes do dano cerebrovascular, o que exige cuidados redobra$\operatorname{dos}^{15,16}$.

A média do número de dias em acompanhamento com a fisioterapia e o número de atendimentos de fisioterapia recebidos pelos pacientes foram de 7 dias e 8 sessóes, respectivamente. Esses achados representam um tempo médio de reabilitação mínimo e talvez ainda não ideal para melhora das condiçôes clínicas, considerando-se que nos seis primeiros meses pós-lesão os indivíduos possuem potencial capacidade em reduzir seus déficits ${ }^{19,20}$ e com isso o tratamento fisioterápico deve ser intensivo, principalmente na fase inicial pós-acometimento. Portanto, faz-se necessário, e, seria ideal, a implementação de serviços que acompanhem o indivíduo em um processo reabilitativo mais extenso, oferecendo todo suporte e tratamento ao paciente logo após a alta hospitalar para continuidade dos cuidados.

O AVC é responsável por grande parte das internaçóes e mortalidade dos idosos ${ }^{12}$, podendo causar na grande maioria dos indivíduos comorbidades ou algum tipo de deficiência, seja parcial ou completa, o que pode impactar no quadro clínico e estado de saúde ${ }^{4,23}$. Nesse estudo, os resultados mostraram que durante a internação hospitalar na unidade de AVC a grande maioria dos pacientes - 93\% não precisaram passar por procedimentos como ventilação mecânica, VNI ou aspiração nasotraqueal. O número de óbitos na unidade foi de apenas $2 \%$ e 4\% dos pacientes tiveram de ser transferidos para o CTI devido complicaçôes clínicas. Esses resultados podem refletir a efetividade do tratamento fisioterápico em evitar complicações respiratórias, além dos cuidados intensivos de toda a equipe de profissionais do setor para com os idosos internados.

Portanto, a partir dos achados, infere-se que a presença de uma unidade de AVC hospitalar, que conte com uma equipe multiprofissional e um serviço de reabilitação eficaz, possa evitar complicações advindas da lesão cerebrovascular e otimizar o tratamento oferecido aos paciente idosos, que são geralmente a maioria dos indivíduos acometidos por AVC.

Os resultados desse estudo podem contribuir para o planejamento e intervenção adequada de medidas reabilitadoras à pacientes idosos pós-AVC agudo e otimização dos serviços de unidades de AVC. Ressalta-se que as fichas de avaliação utilizadas para análise das variáveis desta pesquisa foram as que constavam no serviço de fisioterapia da unidade de AVC do Hospital Risoleta Tolentino Neves e, ademais, salienta-se que as fichas analisadas parecem carecer da inclusão de testes padronizados pela literatura para classificação das variáveis motora-funcionais. Evidenciamos também que os possíveis ganhos motores-funcionais discutidos, devido às particularidades dos dados, não puderam passar por uma análise estatística no qual indicasse a real significância dos achados encontrados. Frisamos ainda que cada setor de reabilitação de centros de internação específicos para cuidados com o paciente na fase aguda do AVC pode possuir um protocolo de avaliação diferente do que foi utilizado nesse estudo.

É necessário mais estudos que caracterizem pacientes em unidades de AVC e que mostrem a importância de se investigar os reais ganhos motores advindos da reabilitação em tal população, além da investigação dos possíveis fatores que interferem no tempo médio entre o ictus e a internação hospitalar. 


\section{CONCLUSÃO}

Evidenciou-se nesse estudo uma média de idade dos idosos internados de 73 anos, sendo que a distribuição do acometimento em ambos os sexos foi relativamente proporcional, com ligeira prevalência em homens. $\mathrm{O}$ AVC isquêmico foi o tipo mais frequente com $85 \%$ dos casos. Os idosos da amostra foram acompanhados pela equipe de reabilitação da unidade de AVC e receberam um número médio de sessóes de fisioterapia de 8 atendimentos. Observou-se que 34\% dos indivíduos tiveram o membro superior classificado como sem déficit motor na avaliação fisioterápica inicial, sendo que esse valor aumentou para 39\% após a avaliação fisioterápica final. Concomitantemente os que tiveram o membro inferior classificado como sem déficit motor passou de 37\% para $43 \%$, respectivamente. Foram observados ainda ganhos nas variáveis motora-funcionais nas classificaçôes CT sem apoio, equilíbrio estático bom e deambulação sem apoio no momento da avaliação fisioterápica final. Além disso, apenas $2 \%$ do total de idosos internados na unidade vieram a óbito e $4 \%$ tiveram de ser transferidos para o CTI. Os resultados desse estudo podem contribuir para o planejamento e intervenção adequada de medidas reabilitadoras à pacientes idosos pós-AVC e otimização dos serviços e metas terapêuticas a serem almejadas em unidades de AVC, além de reforçar a importância dos cuidados clínicos intensivos durante a fase aguda da doença.

\section{REFERÊNCIAS}

1.Freitas EV, Ligia PY. Tratado de geriatria e gerontologia. $3^{a}$ Ed. Rio de Janeiro: Guanabara Koogan, 2011,1750p.

2.Ingall T. Stroke - incidence, mortality, morbidity and risk. J Insur Med 2004;36:143-52.

3.Broeks JG, Lankhorst GJ, Runping K, Prevo AJ. The long-term outcome of arm function after stroke: results of a follow-up study. Disabil Rehabil 1999;21:357-64.

4.Hopman WM, Verner J. Quality of life during and after inpatient stroke rehabilitation. Stroke 2003;34:801-5. http://dx.doi.org/10.1161/01. STR.0000057978.15397.6F

5.Kon K, Chua KSG, Tow AP. Clinical characteristics and functional outcome of stroke patients 75 years old and older. Phys Med Rehabil 1998;79:1535-9. 6.Sandin KJ, Smith BS. The measure of balance in sitting in stroke rehabilitation prognosis. Stroke 1990;21:82-6. http://dx.doi.org/10.1016/S00032993(98)90415-4

7.Kwakkel G, Wagenaar RC, Kollen BJ, Lankhorst GJ. Predicting disability in stroke: A critical review of the literature. Age Ageing 1996;25:479-89. http:// dx.doi.org/10.1093/ageing/25.6.479

8.Loewen SC, Anderson BA. Predictors of stroke outcome using objective measurement scales. Stroke 1990;21:78-81. http://dx.doi.org/10.1161/01. $\underline{\text { STR.21.1.78 }}$

9.Duarte E, Marco E, Muniesa JM, Belmont R, Diaz P, Tejero M, et al. Trunk control test as a functional predictor in stroke patients. J Rehabil Med 2003;34:267-72.

10.Denti L, Agosti M, Franceschini M. Outcome predictors of rehabilitation for first stroke in the elderly. Eur J Phys Rehabil Med 2008;44:3-11.

11.Bagg S, Pombo AP, Hopman W. Effect of age on functional outcomes after stroke rehabilitation. Stroke 2002;33:179-85. http://dx.doi.org/10.1161/ $\underline{\text { hs0102.101224 }}$

12.ArboixA, García-Eroles L, Massons J, Oliveres M, Targa C. Acute stroke in very old people: clinical features and predictors of in-hospital mortality. J Am Geriatr Soc 2000;48:36-41. http://dx.doi.org/10.1161/01. STR.0000077924.71088.02

13.Portaria MS/GM no 665, de 12 de abril de 2012 (endereço na internet). Brasil: Ministério da Saúde (atualizado em: 04/2012; acessado em: 12/2013). Disponível em:

http://bvsms.saude.gov.br/bvs/saudelegis/gm../2012/PRT0665_12_04_2012. html

14.Thommenssen B, Bautz-Holter E, Laake K. Predictors of outcome of rehabilitation of elderly stroke patients in a geriatric ward. Clin Rehabil 1999;13:123-8. http://dx.doi.org/10.1191/026921599666507386

15.Ergeletzis D, Kevorkian CG, Rintala D. Rehabilitation of the older stroke patient: functional outcome and comparison with younger patients. Am J Phys Med Rehabil 2002;81:881-9.

16.Shah MV. Rehabilitation of the Older Adult with Stroke. Clin Geriatr Med 2006;22:469-89. http://dx.doi.org/10.1016/j.cger.2005.12.012

17.Schuster RC, Polese JC, Silva SLA, Perin V, Seben YP. Caracterizaçáo de internaçóes hospitalares por acidente vascular encefálico na cidade de Passo Fundo - RS. Con Scientiae Saúde 2009;8:581-5.

18.Carvalho JJ, Alves MB, Viana GA, Machado CB, dos Santos BF, Kanamura AH, et al. Stroke epidemiology, patterns of management, and outcomes in Fortaleza, Brazil: a hospital-based multicenter prospective study. Stroke 2011;42:3341-6. http://dx.doi.org/10.1161/STROKEAHA.111.626523 19.European Stroke Organization (ESO). Guidelines for Management of Ischaemic Stroke and Transient Ischaemic Attack. Cerebrovas Dis 2008;25:457507. http://dx.doi.org/10.1159/000131083

20.Shepherd R, Carr J. Reabilitação Neurológica: otimizando o desempenho motor. Barueri: Manole, 2008, 384p.

21.Ashburn A. Physical Recovery Following Stroke. Physiotherapy 1997;83:480-90. http://dx.doi.org/10.1016/S0031-9406(05)65636-2

22.Gray CS, French JM, Bates D, Cartlidge NEF, James OFW, Venables G. Motor Recovery Following Acute Stroke. Age and Ageing 1990;19:179-84. http://dx.doi.org/10.1093/ageing/19.3.179

23.Kwon S, Hartzema AG, Ducan PW, Min-Lai S. Disability measures in stroke: relationship among the Barthel Index, the Functional Independence Measure, and the Modified Rankin Scale. Stroke 2004;35:918-23. http:// dx.doi.org/10.1161/01.STR.0000119385.56094.32 